\title{
Lipschitz equivalence of graph-directed fractals
}

\author{
by
}

\author{
Ying Xiong (Guangzhou) and Lifeng Xi (Ningbo)
}

\begin{abstract}
This paper studies the geometric structure of graph-directed sets from the point of view of Lipschitz equivalence. It is proved that if $\left\{E_{i}\right\}_{i}$ and $\left\{F_{j}\right\}_{j}$ are dust-like graph-directed sets satisfying the transitivity condition, then $E_{i_{1}}$ and $E_{i_{2}}$ are Lipschitz equivalent, and $E_{i}$ and $F_{j}$ are quasi-Lipschitz equivalent when they have the same Hausdorff dimension.
\end{abstract}

1. Introduction. Two metric spaces $\left(A, \mathrm{~d}_{A}\right)$ and $\left(B, \mathrm{~d}_{B}\right)$ are called Lipschitz equivalent, denoted by $A \simeq B$, if there exists a bijection $f: A \rightarrow B$ satisfying

$$
c^{-1} \mathrm{~d}_{A}(x, y) \leq \mathrm{d}_{B}(f(x), f(y)) \leq c \mathrm{~d}_{A}(x, y) \quad \text { for all } x, y \in A,
$$

where $c \geq 1$ is a constant.

One of interesting topics in fractal geometry is to classify fractals under Lipschitz equivalence since bi-Lipschitz mappings preserve many "fractal properties" of sets. Many works have been devoted to the related topics. Cooper and Pignataro [1], Falconer and Marsh [4, 5], David and Semmes [2], $\mathrm{Xi}[10,11]$ studied the shape of Cantor sets, nearly Lipschitz equivalence, BPI equivalence and quasi-Lipschitz equivalence. Recently, Xi et al. [8, 13, 14] studied Lipschitz equivalence of self-similar sets.

It is well-known that $E \simeq F$ implies $\operatorname{dim}_{\mathrm{H}} E=\operatorname{dim}_{\mathrm{H}} F$, where $\operatorname{dim}_{\mathrm{H}}$ denotes the Hausdorff dimension. For quasi-self-similar circles, Falconer and Marsh [4] pointed out that two quasi-self-similar circles have the same Hausdorff dimension if and only if they are Lipschitz equivalent.

Then a natural question is to characterize the Lipschitz equivalence for self-similar sets with the same Hausdorff dimension. For a family of similitudes $\left\{S_{i}: \mathbb{R}^{m} \rightarrow \mathbb{R}^{m}\right\}_{i=1}^{n}$, suppose $E=\bigcup_{i} S_{i}(E)$ is a self-similar set [6]. We say $E$ is dust-like [5] if $\bigcup_{i} S_{i}(E)$ is a disjoint union. A number $r$ is the ratio

2000 Mathematics Subject Classification: Primary 28A80.

Key words and phrases: fractal, graph-directed sets, Lipschitz equivalence, quasi-Lipschitz equivalence.

Lifeng $\mathrm{Xi}$ is the corresponding author. 
of similitude of $S$, if $|S(x)-S(y)|=r|x-y|$ for all $x, y \in \mathbb{R}^{m}$. Fix a ratio set $\mathcal{R}=\left\{r_{i}\right\}_{i=1}^{n}$, and let $\mathcal{M}_{\mathcal{R}}$ be the collection of dust-like self-similar sets defined by $\mathcal{M}_{\mathcal{R}}=\left\{E=\bigcup_{i=1}^{n} S_{i}(E): E\right.$ is dust-like and $S_{i}$ has ratio $r_{i}$ for all $\left.i\right\}$. Suppose $\mathcal{R}=\left\{r_{i}\right\}_{i=1}^{n}$ and $\mathcal{T}=\left\{t_{j}\right\}_{j=1}^{m}$ are ratio sets with $\sum_{i} r_{i}^{s}=\sum_{j} t_{j}^{s}=1$. Given $\mathcal{T}=\left\{t_{j}\right\}_{j=1}^{m}$, an algorithm is constructed in [12] to calculate every ratio set $\mathcal{R}$ satisfying $\mathcal{M}_{\mathcal{R}} \simeq \mathcal{M}_{\mathcal{T}}$. It is proved in [5] that if $\mathcal{M}_{\mathcal{R}} \simeq \mathcal{M}_{\mathcal{T}}$, then $\mathbb{Q}\left(r_{1}^{s}, \ldots, r_{n}^{s}\right)=\mathbb{Q}\left(t_{1}^{s}, \ldots, t_{m}^{s}\right)$ and there are positive integers $p, q$ such that $\operatorname{sgp}\left(r_{1}^{p}, \ldots, r_{n}^{p}\right) \subset \operatorname{sgp}\left(t_{1}, \ldots, t_{m}\right), \operatorname{sgp}\left(t_{1}^{q}, \ldots, t_{m}^{q}\right) \subset \operatorname{sgp}\left(r_{1}, \ldots, r_{m}\right)$, where $\operatorname{sgp}\left(a_{1}, \ldots, a_{k}\right)$ is the multiplicative semigroup generated by $\left\{a_{1}, \ldots, a_{k}\right\}$. The following example follows from this necessary condition (see also [3, Proposition 8.9]): Let $C$ be the middle-third Cantor set, and $F=\beta F \cup[\beta F+$ $(1-\beta) / 2] \cup[\beta F+(1-\beta)]$ the self-similar set with $\beta=3^{-\log 3 / \log 2}$. Then $C$ and $F$ have the same dimension $\log 2 / \log 3$, but are not Lipschitz equivalent.

If self-similar sets are not dust-like, for example self-similar arcs, then the issue of their Lipschitz equivalence is complicated. It is proved in [9] that if two self-similar arcs are quasi-arcs with the same Hausdorff dimension, then they are Lipschitz equivalent. [9] also constructs two self-similar $\operatorname{arcs} \gamma_{1}$ and $\gamma_{2}$ such that $\operatorname{dim}_{\mathrm{H}} \gamma_{1}=\operatorname{dim}_{\mathrm{H}} \gamma_{2}$ and $\gamma_{1} \nsucceq \gamma_{2}$. Other overlapping cases, for example the $\{1,3,5\}-\{1,4,5\}$ problem and its generalizations, are studied in $[8,13,14]$.

In this paper, we study the geometric structure of graph-directed sets, which generalizes the notion of self-similar sets, from the point of view of Lipschitz equivalence. For convenience, we recall the definition of graphdirected sets (see [7]).

Definition 1. Let $G=(\mathcal{V}, \mathcal{E})$ be a directed graph with vertex set $\mathcal{V}$ and directed-edge set $\mathcal{E}$. Suppose that for each edge $e \in \mathcal{E}$, there is a corresponding similitude $T_{e}: \mathbb{R}^{n} \rightarrow \mathbb{R}^{n}$ of ratio $t_{e} \in(0,1)$. We also assume the transitivity condition: for any vertex pair $(i, j) \in \mathcal{V} \times \mathcal{V}$, there is a sequence of $k(i, j)$ edges $\left(e_{1}, \ldots, e_{k(i, j)}\right)$ which form a directed path from vertex $i$ to vertex $j$. The graph-directed sets on $G$ with contracting similitudes $\left\{T_{e}\right\}_{e \in \mathcal{E}}$ are non-empty compact subsets $\left\{E_{i}\right\}_{i \in \mathcal{V}}$ of $\mathbb{R}^{n}$ satisfying

$$
E_{i}=\bigcup_{j \in \mathcal{V}} \bigcup_{e \in \mathcal{E}_{i, j}} T_{e}\left(E_{j}\right) \quad \text { for } i \in \mathcal{V}
$$

where $\mathcal{E}_{i, j}$ is the set of edges starting at $i$ and ending at $j$. In particular, if (1.1) is a disjoint union for each $i \in \mathcal{V}$, we say that $\left\{E_{i}\right\}_{i \in \mathcal{V}}$ are dust-like graph-directed sets on $(\mathcal{V}, \mathcal{E})$.

REMARK 1. The graph with respect to a self-similar set only contains one vertex.

Now we state our first result about the Lipschitz equivalence between dust-like graph-directed sets. 
TheOREM 1. Let $\left\{E_{i}\right\}_{i \in \mathcal{V}}$ be dust-like graph-directed sets on $G=(\mathcal{V}, \mathcal{E})$ satisfying the transitivity condition (see Definition 1 ). Then for all $i, j \in \mathcal{V}$,

$$
E_{i} \simeq E_{j}
$$

The following classical result in [1] can also be considered as a corollary of the above theorem.

Corollary 1. Suppose $E \subset \mathbb{R}^{m}$ is a dust-like self-similar set. Let $F=\bigcup_{i=1}^{k} g_{i}(E)$ be a disjoint union with a family of similitudes $\left\{g_{i}: \mathbb{R}^{m} \rightarrow\right.$ $\left.\mathbb{R}^{m}\right\}_{i=1}^{k}$. Then $E$ and $F$ are Lipschitz equivalent.

By the example mentioned above, it is difficult to find a bi-Lipschitz bijection between self-similar sets. However, we can construct some bijection which satisfies the "quasi-Lipschitz" condition (see Definition 2) between two dust-like graph-directed sets of equal dimension. The definition below was introduced by Xi [11].

Definition 2. Two compact sets $E$ and $F$ of Euclidean spaces are said to be quasi-Lipschitz equivalent if there is a bijection $f: E \rightarrow F$ such that for every $\varepsilon>0$, there exists $\delta>0$ satisfying

$$
\left|\frac{\log |f(x)-f(y)|}{\log |x-y|}-1\right|<\varepsilon
$$

whenever $x, y \in E$ with $0<|x-y|<\delta$.

The quasi-Lipschitz equivalence is stronger than "nearly Lipschitz equivalence" ([5]) and weaker than "Lipschitz equivalence". There are some related results: Suppose $E, F$ are dust-like $C^{1}$ self-conformal sets in Euclidean spaces. Then $\operatorname{dim}_{\mathrm{H}} E=\operatorname{dim}_{\mathrm{H}} F$ if and only if $E$ and $F$ are nearly Lipschitz equivalent $([5,10])$. In fact, $\operatorname{dim}_{\mathrm{H}} E=\operatorname{dim}_{\mathrm{H}} F$ if and only if $E$ and $F$ are quasi-Lipschitz equivalent ([11]).

Now suppose two graph-directed sets have the same Hausdorff dimension; a question is to characterize the quasi-Lipschitz equivalence between them, although they may not be Lipschitz equivalent. We can state our second result.

THEOREM 2. Let $\left\{E_{i}\right\}_{i=1}^{m}$ and $\left\{F_{j}\right\}_{j=1}^{n}$ be dust-like graph-directed sets satisfying the transitivity condition. If $\operatorname{dim}_{\mathrm{H}} E_{i}=\operatorname{dim}_{\mathrm{H}} F_{j}$, then $E_{i}$ and $F_{j}$ are quasi-Lipschitz equivalent.

This paper is organized as follows. Section 2 brings the proofs of Theorem 1 and Corollary 1. In Section 3, the proof of Theorem 2 is provided.

2. The proof of Theorem 1. In this section, we always assume that the sets $\left\{E_{i}\right\}_{i \in \mathcal{V}}$ are dust-like graph-directed sets on $G=(\mathcal{V}, \mathcal{E})$ satisfying the transitivity condition (see Definition 1). We begin with two lemmas 
which follow immediately from the definitions of dust-like graph-directed sets and the transitivity condition.

Lemma 1. Suppose that $E \in\left\{E_{i}\right\}_{i \in \mathcal{V}}$. Then there are contracting similitudes $S_{0}, S_{1}$ and a compact set $F$ such that

$$
E=S_{0}(E) \cup S_{1}(E) \cup F,
$$

where the union is disjoint.

Lemma 2. Suppose that $E \in\left\{E_{i}\right\}_{i \in \mathcal{V}}$. Then there are non-empty families $\left\{\Gamma_{j}\right\}_{j \in \mathcal{V}}$ consisting of contracting similitudes such that

$$
E=\bigcup_{j \in \mathcal{V}} \bigcup_{S \in \Gamma_{j}} S\left(E_{j}\right)
$$

where the union is disjoint.

We skip the straightforward proofs of the above two lemmas. The lemma below is the key point in the proof of Theorem 1 and may be of interest in itself.

Lemma 3. Suppose that $E \in\left\{E_{i}\right\}_{i \in \mathcal{V}}$. Then for any similitudes $\left\{T_{i}\right\}_{i=0}^{k}$ such that $\left\{T_{i}(E)\right\}_{i=0}^{k}$ are pairwise disjoint,

$$
E \simeq T_{0}(E) \cup T_{1}(E) \cup \cdots \cup T_{k}(E) .
$$

Proof. By induction, it suffices to verify the conclusion for $k=1$, i.e.,

$$
E \simeq T_{0}(E) \cup T_{1}(E) .
$$

By Lemma 1, we have

$$
E=S_{0}(E) \cup S_{1}(E) \cup F,
$$

where the union is disjoint. For a finite word $i_{1} \ldots i_{k} \in\{0,1\}^{k}$, put $S_{i_{1} \ldots i_{k}}=$ $S_{i_{1}} \circ \cdots \circ S_{i_{k}}$, where $S_{w}$ equals the identity mapping if $w$ is the empty word. We also use $1^{k}$ as an abbreviation of $1 \ldots 1$ ( $k$ ones). With this notation,

$$
\begin{aligned}
E & =\left(S_{0} E \cup F\right) \cup\left(S_{10} E \cup S_{1} F \cup S_{11} E\right) \\
& =\left(S_{0} E \cup F\right) \cup\left(S_{10} E \cup S_{1} F\right) \cup\left(S_{110} E \cup S_{11} F \cup S_{111} E\right) \\
& =\bigcup_{k=0}^{\infty}\left(S_{1^{k} 0} E \cup S_{1^{k}} F\right) \cup\{\omega\},
\end{aligned}
$$

where $\omega$ is the fixed point of the similitude $S_{1}$. Consequently, we can write

$$
E=S_{0} E \cup \bigcup_{k=0}^{\infty} S_{1^{k+1} 0} E \cup\left(\bigcup_{k=0}^{\infty} S_{1^{k}} F \cup\{\omega\}\right)=: S_{0} E \cup E^{\prime} \cup F^{\prime},
$$

where $E^{\prime} \cup F^{\prime}=S_{1} E \cup F$, and

$$
T_{0} E \cup T_{1} E=T_{0} E \cup \bigcup_{k=0}^{\infty} T_{1} S_{1^{k} 0} E \cup\left(\bigcup_{k=0}^{\infty} T_{1} S_{1^{k}} F \cup\left\{T_{1} \omega\right\}\right)=: T_{0} E \cup E^{\prime \prime} \cup F^{\prime \prime},
$$


where $E^{\prime \prime} \cup F^{\prime \prime}=T_{1} E$. We define a bijection $f: E \rightarrow T_{0} E \cup T_{1} E$ by

$$
f(x)= \begin{cases}T_{0} S_{0}^{-1}(x) & \text { if } x \in S_{0} E, \\ T_{1} S_{1}^{-1}(x) & \text { if } x \in E^{\prime}=\bigcup_{k=0}^{\infty} S_{1^{k+1} 0} E, \\ T_{1}(x) & \text { if } x \in F^{\prime}=\bigcup_{k=0}^{\infty} S_{1^{k}} F \cup\{\omega\} .\end{cases}
$$

It remains to show that $f$ is bi-Lipschitz.

Since

$$
d\left(S_{0} E, E^{\prime} \cup F^{\prime}\right)>0 \quad \text { and } \quad d\left(T_{0} E, E^{\prime \prime} \cup F^{\prime \prime}\right)>0,
$$

where $d$ is the Hausdorff distance, we only need to consider the restriction of $f$ to $E^{\prime} \cup F^{\prime}$ (the corresponding image is $E^{\prime \prime} \cup F^{\prime \prime}$ ). Suppose $s_{0}, s_{1}, t_{1}$ are the ratios of $S_{0}, S_{1}, T_{1}$, respectively. Put

$$
\Delta:=\min \left\{d\left(S_{0} E, S_{1} E\right), d\left(S_{0} E, F\right), d\left(S_{1} E, F\right)\right\}>0 .
$$

For $x \in E^{\prime}$ and $y \in F^{\prime}$, suppose that $x \in S_{1^{m+1}{ }_{0}} E=S_{1^{m+1}}\left(S_{0} E\right)$ with $m \geq 0$ and $y \in S_{1^{k}} F$ with $k \geq 0$ or $k=\infty$. Here $S_{1 \infty} F=\{\omega\}$. Then $f(x) \in T_{1} S_{1^{m} 0} E$ and $f(y) \in T_{1} S_{1^{k}} F$. Let $|E|$ be the diameter of $E$. Then

$$
\begin{aligned}
& s_{1}^{\min (m+1, k)} \Delta \leq|x-y| \leq s_{1}^{\min (m+1, k)}|E|, \\
& t_{1} s_{1}^{\min (m, k)} \Delta \leq|f(x)-f(y)| \leq t_{1} s_{1}^{\min (m, k)}|E| .
\end{aligned}
$$

Therefore, for any $x \in E^{\prime}$ and $y \in F^{\prime}$,

$$
\begin{aligned}
\frac{t_{1} \Delta}{|E|} & \leq \frac{s_{1}^{\min (m, k)}}{s_{1}^{\min (m+1, k)}} \frac{t_{1} \Delta}{|E|} \leq \frac{|f(x)-f(y)|}{|x-y|} \\
& \leq \frac{s_{1}^{\min (m, k)}}{s_{1}^{\min (m+1, k)}} \frac{t_{1}|E|}{\Delta} \leq \frac{t_{1}|E|}{s_{1} \Delta} .
\end{aligned}
$$

Proof of Theorem 1. Let $\left\{\Psi_{j}\right\}_{j \in \mathcal{V}}$ be a family of similitudes such that the sets $\left\{\Psi_{j}\left(E_{j}\right)\right\}_{j \in \mathcal{V}}$ are pairwise disjoint. Let $E \in\left\{E_{i}\right\}_{i \in \mathcal{V}}$ and $\left\{\Gamma_{j}\right\}_{j \in \mathcal{V}}$ be as in Lemma 2. Then

$$
E=\bigcup_{j \in \mathcal{V}} \bigcup_{S \in \Gamma_{j}} S\left(E_{j}\right)
$$

where the union is disjoint. According to Lemma 3, for any $j \in \mathcal{V}$,

$$
\bigcup_{S \in \Gamma_{j}} S\left(E_{j}\right) \simeq \Psi_{j}\left(E_{j}\right)
$$

Then for any $E \in\left\{E_{i}\right\}_{i \in \mathcal{V}}$,

$$
E=\bigcup_{j \in \mathcal{V}} \bigcup_{S \in \Gamma_{j}} S\left(E_{j}\right) \simeq \bigcup_{j \in \mathcal{V}} \Psi_{j}\left(E_{j}\right),
$$

which implies $E_{i} \simeq E_{j}$ for all $i, j \in \mathcal{V}$. 
Corollary 1 follows from Lemma 3, since any self-similar set has a special graph-directed construction with its graph containing only one point. We also give another proof by using Theorem 1 as follows.

Proof of Corollary 1. By induction, it suffices to show $E \simeq g_{1}(E) \cup g_{2}(E)$ for the dust-like self-similar set $E=\bigcup_{i=1}^{m} S_{i}(E)$, which is a disjoint union. Since $g_{1}(E) \simeq g_{2}(E) \simeq S_{1}(E) \simeq S_{2}(E) \simeq E$, we only need to prove $E \simeq$ $S_{1}(E) \cup S_{2}(E)$. If $m=2$, then $E=S_{1}(E) \cup S_{2}(E)$. Without loss of generality, we assume that $m \geq 3$.

Let $\varrho_{i}$ be the ratio of $S_{i}$ for any $i$. Take $\left\{r_{i}\right\}_{i=2}^{m-1}$ such that

$$
\max \left\{\varrho_{1}, \ldots, \varrho_{m}\right\}<r_{2}<\cdots<r_{m-1}<1 .
$$

Let $E_{1}=E$ and $E_{k}=r_{k}^{-1}\left[S_{1}(E) \cup \cdots \cup S_{k}(E)\right]$ for $1<k<m$. Then we get a dust-like graph-directed construction satisfying the transitivity condition:

$$
\begin{aligned}
& E_{2}=r_{2}^{-1}\left[S_{1}\left(E_{1}\right) \cup S_{2}\left(E_{1}\right)\right], \\
& E_{k}=r_{k}^{-1}\left[\left(r_{k-1} E_{k-1}\right) \cup\left(S_{k} E_{1}\right)\right] \quad \text { for } k \in \mathbb{N} \cap(2, m-1], \\
& E_{1}=r_{m-1} E_{m-1} \cup S_{m}\left(E_{1}\right) .
\end{aligned}
$$

Therefore, it follows from Theorem 1 that $E_{2} \simeq E_{1}$. Here $E_{1}=E$ and $E_{2} \simeq S_{1}(E) \cup S_{2}(E)$, which implies $E \simeq S_{1}(E) \cup S_{2}(E)$.

3. The proof of Theorem 2. Let $\left\{E_{i}\right\}_{i \in \mathcal{V}}$ be dust-like graph-directed sets satisfying the transitivity condition. Denote by $\left\{t_{e}\right\}_{e \in \mathcal{V}}$ the ratio set of $\left\{E_{i}\right\}_{i \in \mathcal{V}}$. Write $t_{*}=\min \left\{t_{e}: e \in \mathcal{V}\right\}>0$. By iterating (1.1) we can obtain the following lemma.

Lemma 4. Suppose that $0<r<t_{*}$. Then there are families $\left\{\Gamma_{i, j}^{r}\right\}_{i, j \in \mathcal{V}}$ of similitudes such that

$$
E_{i}=\bigcup_{j \in \mathcal{V}} \bigcup_{S \in \Gamma_{i, j}^{r}} S\left(E_{j}\right) \quad \text { for all } i, j \in \mathcal{V},
$$

where the union is disjoint, and $r(S) \in\left(t_{*} r, r\right]$ for any $S \in \bigcup_{i, j \in \mathcal{V}} \Gamma_{i, j}^{r}$.

Instead of proving Theorem 2 directly, we will prove the following proposition.

Proposition 1. Let $\left\{E_{i}\right\}_{i \in \mathcal{V}}$ be dust-like graph-directed sets satisfying the transitivity condition. Suppose $\Sigma_{2}=\{0,1\}^{\mathbb{N}}$ is the symbolic space of two letters equipped with the usual distance

$$
d\left(\sigma, \sigma^{\prime}\right)=2^{-\min \left\{k: \sigma_{k} \neq \sigma_{k}^{\prime}\right\}} \quad \text { for } \sigma \neq \sigma^{\prime} .
$$

If $E \in\left\{E_{i}\right\}_{i \in \mathcal{V}}$, then there is a bijection $f: E \rightarrow \Sigma_{2}$ such that for every $\varepsilon>0$, there exists $\delta>0$ satisfying 


$$
\left|\frac{\log d(f(x), f(y))}{\operatorname{dim}_{\mathrm{H}} E \cdot \log |x-y|}-1\right|<\varepsilon
$$

whenever $x, y \in E$ with $0<|x-y|<\delta$.

As in [11], we can see that Theorem 2 is a corollary of Proposition 1. In fact, for dust-like graph-directed sets $\left\{E_{i}\right\}_{i}$ and $\left\{F_{j}\right\}_{j}$ satisfying the transitivity condition, if $E \in\left\{E_{i}\right\}_{i}$ and $F \in\left\{F_{j}\right\}_{j}$ have the same Hausdorff dimension $s$, then it follows from Proposition 1 that there are bijections $f: E \rightarrow \Sigma_{2}$ and $g: F \rightarrow \Sigma_{2}$ such that

$$
\frac{\log d\left(f\left(x_{1}\right), f\left(x_{2}\right)\right)}{s \log \left|x_{1}-x_{2}\right|} \rightarrow 1, \quad \frac{\log d\left(g\left(y_{1}\right), g\left(y_{2}\right)\right)}{s \log \left|y_{1}-y_{2}\right|} \rightarrow 1
$$

uniformly whenever $\left|x_{1}-x_{2}\right|,\left|y_{1}-y_{2}\right| \rightarrow 0$. Then $g^{-1} \circ f: E \rightarrow F$ is a bijection satisfying (1.2).

Proof of Proposition 1. We begin with some notation for symbolic systems. Given $w=w_{1} \ldots w_{k} \in\{0,1\}^{k}$ and $w^{\prime}=w_{1}^{\prime} \ldots w_{k^{\prime}}^{\prime} \in\{0,1\}^{k^{\prime}}$, write $w * w^{\prime}:=w_{1} \ldots w_{k} w_{1}^{\prime} \ldots w_{k^{\prime}}^{\prime} \in\{0,1\}^{k+k^{\prime}}$, and let $[w]$ denote the cylinder with respect to $w$, i.e.,

$$
[w]:=\left\{\sigma \in \Sigma_{2}: \sigma_{1} \ldots \sigma_{k}=w\right\} .
$$

Given $w$, we can split $[w]$ into two cylinders $[w * 0]$ and $[w * 1]$.

Let $r_{k}=t_{*} \cdot 2^{-k}\left(<t_{*}\right)$ for $k \geq 1$. Then it follows from Lemma 4 that there are corresponding families $\left\{\Gamma_{i, j}^{r_{k}}\right\}_{i, j \in \mathcal{V}}$ for all $k \geq 1$ such that $E_{i}=\bigcup_{j \in \mathcal{V}} \bigcup_{S \in \Gamma_{i, j}^{r_{k}}} S\left(E_{j}\right)$ with $r(S) \in\left(t_{*} r_{k}, r_{k}\right]$ for any $S \in \Gamma_{i, j}^{r_{k}}$. Write

$$
\Xi_{i}^{k}=\bigcup_{j \in \mathcal{V}} \Gamma_{i, j}^{r_{k}}
$$

We will estimate $\# \Xi_{i}^{k}$, the cardinality of $\Xi_{i}^{k}$. Let $\bar{M}=\max \left\{\mathcal{H}^{s}\left(E_{i}\right)\right.$ : $i \in \mathcal{V}\}$ and $\underline{M}=\min \left\{\mathcal{H}^{s}\left(E_{i}\right): i \in \mathcal{V}\right\}$, where $s=\operatorname{dim}_{\mathrm{H}} E_{i}$ for any $i \in \mathcal{V}$. Notice that $0<\underline{M} \leq \bar{M}<\infty$. It follows from Lemma 4 that

$$
\mathcal{H}^{s}\left(E_{i}\right)=\sum_{j \in \mathcal{V}, S \in \Gamma_{i, j}^{r_{k}}} \mathcal{H}^{s}\left(S\left(E_{j}\right)\right),
$$

where $r(S) \in\left(t_{*} r_{k}, r_{k}\right]$, which implies

$$
\left(t_{*} r_{k}\right)^{s} \underline{M} \leq\left(t_{*} r_{k}\right)^{s} \mathcal{H}^{s}\left(E_{j}\right) \leq \mathcal{H}^{s}\left(S\left(E_{j}\right)\right) \leq r_{k}^{s} \mathcal{H}^{s}\left(E_{j}\right) \leq r_{k}^{s} \bar{M} .
$$

Therefore,

$$
\frac{\underline{M}}{r_{k}^{s} \bar{M}} \leq \# \Xi_{i}^{k} \leq \frac{\bar{M}}{t_{*}^{s} r_{k}^{s} \underline{M}}
$$


For each $\Xi_{i}^{k}$, there is an integer $n(i, k)$ such that $2^{n(i, k)} \leq \# \Xi_{i}^{k}<$ $2^{n(i, k)+1}$, which implies

$$
\frac{t_{*}^{s} \underline{M}}{2 \bar{M}} r_{k}^{s} \leq 2^{-[n(i, k)+1]} \leq 2^{-n(i, k)} \leq \frac{2 \bar{M}}{\underline{M}} r_{k}^{s} .
$$

By splitting \# $\Xi_{i}^{k}-2^{n(i, k)}$ cylinders with respect to the word of length $n(i, k)$, we can find a family $\Sigma_{i}^{k}$ consisting of finite words such that

(i) any word in $\Sigma_{i}^{k}$ is of length $n(i, k)$ or $n(i, k)+1$;

(ii) $\Sigma_{2}=\bigcup_{w \in \Sigma_{i}^{k}}[w]$ is a disjoint union;

(iii) $\# \Sigma_{i}^{k}=\# \Xi_{i}^{k}$.

Thus, we can find a one-to-one mapping $\pi_{i}^{k}: \Xi_{i}^{k} \rightarrow \Sigma_{i}^{k}$ for all $k \geq 1$ and $i \in \mathcal{V}$.

Now, for each $E \in\left\{E_{i}\right\}_{i \in \mathcal{V}}$, we can construct a bijection $f: E \rightarrow \Sigma_{2}$. Let $x \in E$; according to the construction of graph-directed sets, there are corresponding $i_{k} \in \mathcal{V}$ and $S_{k} \in \Gamma_{i_{k}, i_{k+1}}^{r_{k}}\left(\subset \Xi_{i_{k}}^{k}\right)$ for all $k \geq 1$ such that

$$
E=E_{i_{1}} \quad \text { and } \quad x \in S_{1} \circ \cdots \circ S_{k}\left(E_{i_{k+1}}\right) \quad \text { for } k \geq 1 \text {. }
$$

The bijection $f: E \rightarrow \Sigma_{2}$ is defined by $f(x)=\sigma$ where for all $k \geq 1$,

$$
\sigma \in\left[\pi_{i_{1}}^{1}\left(S_{1}\right) * \cdots * \pi_{i_{k}}^{k}\left(S_{k}\right)\right] .
$$

To show that $f$ is as desired, we need some more notation. Put $\bar{D}=$ $\max _{i \in \mathcal{V}}\left\{\left|E_{i}\right|\right\}$; let $\underline{D}_{i}=\min \left\{d\left(T_{e}\left(E_{j}\right), T_{e^{\prime}}\left(E_{j^{\prime}}\right)\right): e \neq e^{\prime}\right.$ with $e \in \mathcal{E}_{i, j}$, $\left.e^{\prime} \in \mathcal{E}_{i, j^{\prime}}\right\}$ and $\underline{D}=\min _{i \in \mathcal{V}}\left\{\underline{D}_{i}\right\}>0$.

Without loss of generality, suppose $x, y \in E$ are distinct points such that $x \in S_{1} \circ \cdots \circ S_{N-1} \circ S_{N}\left(E_{i_{N+1}}\right), \quad y \in S_{1} \circ \cdots \circ S_{N-1} \circ S_{N}^{\prime}\left(E_{i_{N+1}^{\prime}}\right)$,

where $S_{N}, S_{N}^{\prime} \in \Xi_{i_{N}}^{N}$ with $S_{N} \neq S_{N}^{\prime}$. Then

$$
d\left(S_{N}\left(E_{i_{N+1}}\right), S_{N}^{\prime}\left(E_{i_{N+1}^{\prime}}\right)\right) \geq t_{*} r_{N} \underline{D} .
$$

It follows from Lemma 4 and (3.3) that

$$
t_{*}^{N} r_{1} \cdots r_{N} \cdot \underline{D} \leq|x-y| \leq r_{1} \cdots r_{N-1} \cdot \bar{D} .
$$

On the other hand,

$$
\begin{aligned}
& f(x) \in\left[\pi_{i_{1}}^{1}\left(S_{1}\right) * \cdots * \pi_{i_{N-1}}^{N-1}\left(S_{N-1}\right) * \pi_{i_{N}}^{N}\left(S_{N}\right)\right], \\
& f(y) \in\left[\pi_{i_{1}}^{1}\left(S_{1}\right) * \cdots * \pi_{i_{N-1}}^{N-1}\left(S_{N-1}\right) * \pi_{i_{N}}^{N}\left(S_{N}^{\prime}\right)\right],
\end{aligned}
$$

where $\pi_{i_{N}}^{N}\left(S_{N}\right) \neq \pi_{i_{N}}^{N}\left(S_{N}^{\prime}\right)$. Together with (3.1), (3.2) and condition (i) about $\Sigma_{i}^{k}$, we get

$$
\left(\frac{t_{*}^{s} \underline{M}}{2 \bar{M}}\right)^{N}\left(r_{1} \cdots r_{N}\right)^{s} \leq d(f(x), f(y)) \leq\left(\frac{2 \bar{M}}{\underline{M}}\right)^{N-1}\left(r_{1} \cdots r_{N-1}\right)^{s},
$$


where $s=\operatorname{dim}_{\mathrm{H}} E$. In view of (3.4), (3.5) and the fact that $r_{k}=t_{*} \cdot 2^{-k}$, we have $N \rightarrow \infty$ uniformly as $|x-y| \rightarrow 0$, and thus

$$
\frac{\log d(f(x), f(y))}{\log |x-y|} \rightarrow s=\operatorname{dim}_{\mathrm{H}} E \quad \text { uniformly as }|x-y| \rightarrow 0
$$

where

$$
\frac{\log \left(r_{1} \cdots r_{N}\right)}{-\left(N^{2} / 2\right) \log 2} \rightarrow 1
$$

Acknowledgements. This research is supported by National Natural Science Foundation of China (no. 10671180, 10571140, 10571063, 10631040) and Morningside Center of Mathematics.

\section{References}

[1] D. Cooper and T. Pignataro, On the shape of Cantor sets, J. Differential Geom. 28 (1988), 203-221.

[2] G. David and S. Semmes, Fractured Fractals and Broken Dreams. Self-Similar Geometry through Metric and Measure, Oxford Lecture Ser. Math. Appl. 7, Clarendon Press, New York, 1997.

[3] K. J. Falconer, Techniques in Fractal Geometry, Wiley, Chichester, 1997.

[4] K. J. Falconer and D. T. Marsh, Classification of quasi-circles by Hausdorff dimension, Nonlinearity 2 (1989), 489-493.

[5] -, -, On the Lipschitz equivalence of Cantor sets, Mathematika 39 (1992), 223-233.

[6] J. E. Hutchinson, Fractals and self similarity, Indiana Univ. Math. J. 30 (1981), 713-747.

[7] R. D. Mauldin and S. C. Williams, Hausdorff dimension in graph directed constructions, Trans. Amer. Math. Soc. 309 (1988), 811-829.

[8] H. Rao, H. J. Ruan, and L. F. Xi, Lipschitz equivalence of self-similar sets, C. R. Math. Acad. Sci. Paris 342 (2006), 191-196.

[9] Z. Y. Wen and L. F. Xi, Relations among Whitney sets, self-similar arcs and quasiarcs, Israel J. Math. 136 (2003), 251-267.

[10] L. F. Xi, Lipschitz equivalence of self-conformal sets, J. London Math. Soc. (2) 70 (2004), 369-382.

[11] —, Quasi-Lipschitz equivalence of fractals, Israel J. Math. 160 (2007), 1-21.

[12] —, Lipschitz equivalence of dust-like self-similar sets, submitted.

[13] L. F. Xi and H. J. Ruan, Lipschitz equivalence of generalized $\{1,3,5\}-,\{1,4,5\}$ self-similar sets, Sci. China Ser. A 50 (2007), 1537-1551.

[14] L. F. Xi, H. J. Ruan, and Q. L. Guo, Sliding of self-similar sets, ibid. 50 (2007), 351-360.

Department of Mathematics

South China University of Technology

Guangzhou, 510641, P.R. China

E-mail: xiongyng@gmail.com
Institute of Mathematics

Zhejiang Wanli University

Ningbo, Zhejiang, 315100, P.R. China

E-mail: xilifengningbo@yahoo.com 